\title{
Scaling HTM-Supported Database Transactions to Many Cores
}

\author{
Viktor Leis, Alfons Kemper, and Thomas Neumann
}

\begin{abstract}
So far, transactional memory-although a promising technique—suffered from the absence of an efficient hardware implementation. Intel's Haswell microarchitecture introduced hardware transactional memory (HTM) in mainstream CPUs. HTM allows for efficient concurrent, atomic operations, which is also highly desirable in the context of databases. On the other hand HTM has several limitations that, in general, prevent a one-to-one mapping of database transactions to HTM transactions.

In this work we devise several building blocks that can be used to exploit HTM in main-memory databases. We show that HTM allows to achieve nearly lock-free processing of database transactions by carefully controlling the data layout and the access patterns. The HTM component is used for detecting the (infrequent) conflicts, which allows for an optimistic, and thus very low-overhead execution of concurrent transactions. We evaluate our approach on a 4-core desktop and a 28-core server system and find that HTM indeed provides a scalable, powerful, and easy to use synchronization primitive.
\end{abstract}

Index Terms-hardware transactional memory, synchronization, concurrency control, transaction processing

\section{INTRODUCTION}

The support for hardware transactional memory (HTM) in mainstream processors like Intel's Haswell appears like a perfect fit for emerging main-memory database systems like H-Store/VoltDB [1], HyPer [2], SAP HANA [3], SolidDB [4], Microsoft Hekaton [5], etc. Transactional memory [6] is a very intriguing concept that allows for automatic atomic and concurrent execution of arbitrary code. Transactional memory allows for code like this:

\section{transaction \{ \\ $a=a-10$; \\ $b=b+10$; \} \\ Transaction 1}

$$
\begin{array}{r}
\text { transaction }\{ \\
c=c-20 ; \\
\quad a=a+20 ;
\end{array}
$$

Transaction 2
Semantically, this code behaves quite similar to database transactions. The code sections are executed atomically and in isolation from each other. In the case of runtime conflicts (i.e., read/write conflicts or write/write conflicts) a transaction might get aborted, undoing all changes performed so far. The transaction model is a very elegant and well understood idea that is much simpler than the classical alternative, namely finegrained locking. Locking is much more difficult to formulate correctly. Fine-grained locking is error prone and can lead to deadlocks due to differences in locking order. Coarse-grained locking is simpler, but greatly reduces concurrency. Transactional memory avoids this problem by keeping track of read and write sets and thus by detecting conflicts on the memory access level. Starting with the Haswell microarchitecture this is supported by hardware, which offers excellent performance.

Figure 1 sketches the benefits of our HTM-based transaction manager in comparison to other concurrency control

- The authors are with the Database Group, Department of Informatics, Technische Universität München, Garching, Germany.

E-mail: \{lastname\}@in.tum.de

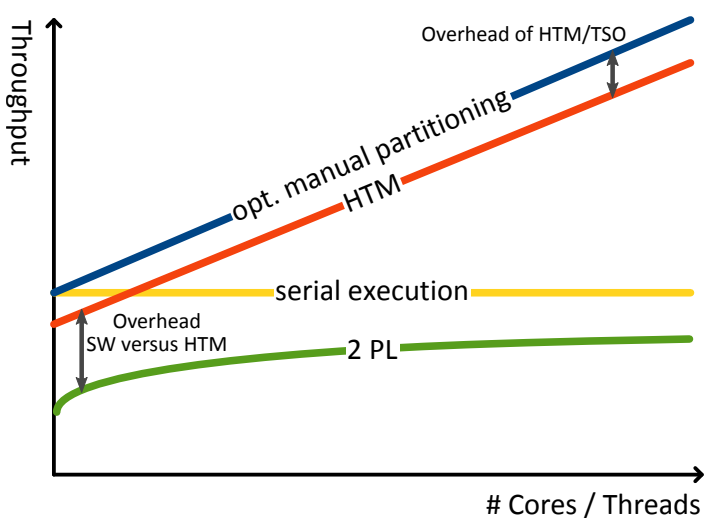

Figure 1. HTM versus $2 \mathrm{PL}$, sequential, partitioned

mechanisms that we investigated. For main-memory database applications the well-known Two Phase Locking scheme was proven to be inferior to serial execution [7]! However, serial execution cannot exploit the parallel compute power of modern multi-core CPUs. Under serial execution, scaling the throughput in proportion to the number of cores would require an optimal partitioning of the database such that transactions do not cross these boundaries. This allows for "embarrassingly" parallel execution-one thread within each partition. Unfortunately, this is often not possible in practice; therefore, the upper throughput curve "opt. manual partitioning" of Figure 1 is only of theoretical nature. HTM, however, comes very close to an optimal static partitioning scheme as its transaction processing can be viewed as an adaptive dynamic partitioning of the database according to the transactional access pattern.

However, transactional memory is no panacea for transaction processing. First, database transactions also require properties like durability, which are beyond the scope of transactional memory. Second, at least the current hardware 


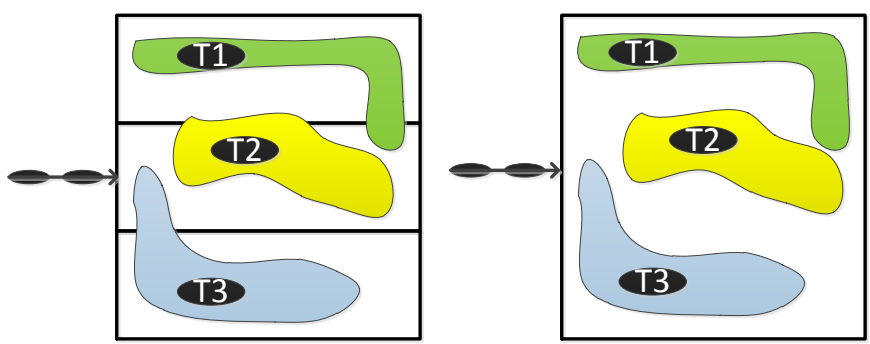

Figure 2. Static partitioning (left), Optimistic concurrency control via HTM resulting in dynamic partitioning (right)

implementations of transactional memory are limited. For the Haswell microarchitecture the scope of a transaction is limited, because the read/write set, i.e., every cache line a transaction accesses, has to fit into the $\mathrm{L} 1$ cache with a capacity of $32 \mathrm{~KB}$. Furthermore, HTM transactions may fail due to a number of unexpected circumstances like collisions caused by cache associativity, hardware interrupts, etc. Therefore, it does not seem to be viable to map an entire database transaction to a single monolithic HTM transaction. In addition, one always needs a "slow path" to handle the pathological cases (e.g., associativity collisions).

We therefore propose an architecture where transactional memory is used as a building block for assembling complex database transactions. Along the lines of the general philosophy of transactional memory we start executing transactions optimistically, using (nearly) no synchronization and thus running at full clock speed. By exploiting HTM we get many of the required checks for free, without complicating the database code, and can thus reach a much higher degree of parallelism than with classical locking or latching. In order to minimize the number of conflicts in the transactional memory component, we carefully control the data layout and the access patterns of the involved operations, which allows us to avoid explicit synchronization most of the time.

Note that we explicitly do not assume that the database is partitioned in any way. In some cases, and in particular for the well-known TPC-C benchmark, the degree of parallelism can be improved greatly by partitioning the database at the schema level (using the warehouse attribute in the case of TPC-C). Such a static partitioning scheme is exemplified on the lefthand side of Figure 2. VoltDB for example makes use of static partitioning for parallelism [1]. But such a partitioning is hard to find in general, and users usually cannot be trusted to find perfect partitioning schemes [8]. In addition, there can always be transactions that cross partition boundaries, as shown by the partition boundary overlapping transactions $\mathrm{T} 1, \mathrm{~T} 2$, and T3 in Figure 2 (left-hand side). These transactions have to be isolated with a serial (or locking-based) approach as the static partitioning scheme cannot guarantee their isolation. If available, we could still exploit partitioning information in our HTM approach, of course, as then conflicts would be even more unlikely. But we explicitly do not assume the presence of such a static partitioning scheme and rely on the implicit adaptive partitioning of the transactions as sketched on the right-hand side of Figure 2.
This article is an extended version of the conference paper [9]. The new Section 3 experimentally evaluates HTM and alternative synchronization mechanisms like latching on a 28-core Haswell system. Furthermore, we devise a set of best practices that allow HTM to scale very well even with a large number of cores.

\section{Transactional Memory}

Traditional synchronization mechanisms are usually implemented using some form of mutual exclusion (mutex). For 2PL, the DBMS maintains a lock structure that keeps track of all currently held locks. As this lock structure is continuously updated by concurrent transactions, the structure itself is protected by one (or more) mutexes [10]. On top of this, the locks themselves provide a kind of mutual exclusion mechanism, and block a transaction if needed.

The serial execution paradigm is even more extreme, there one lock protects the whole database (or the whole partition for partitioned execution). The problem with these locks is that they are difficult to use effectively. In particular, finding the right lock granularity is difficult. Coarse locks are cheap, but limit concurrency. Fine-grained locks allow for more concurrency, but are more expensive and can lead to deadlocks.

For quite some time now, transactional memory is being proposed as an alternative to fine grained locking [6]. The key idea behind transactional memory is that a number of operations can be combined into a transaction, which is then executed atomically. Consider the following small code fragment for transferring money from one account to another account (using GCC syntax):

\section{transfer(from,to,amount) transaction_atomic \{ account[from]-=amount; account[to]+=amount; \\ \}}

The code inside the atomic block is guaranteed to be executed atomically, and in isolation. In practice, the transactional memory observes the read set and write set of transactions, and executes transactions concurrently as long as the sets do not conflict. Thus, transfers can be executed concurrently as long as they affect different accounts, they are only serialized if they touch a common account. This behavior is very hard to emulate using locks. Fine-grained locking would allow for high concurrency, too, but would deadlock if accounts are accessed in opposite order. Transactional memory solves this problem elegantly.

Transactional memory has been around for a while, but has usually been implemented as Software Transactional Memory (STM), which emulated this behavior in software. Although STM does remove the complexity of lock maintenance, it causes a significant slowdown during execution and thus had limited practical impact [11].

\subsection{Hardware Support for Transactional Memory}

This changed with the Haswell microarchitecture from Intel, which offers Hardware Transactional Memory [12]. Note 

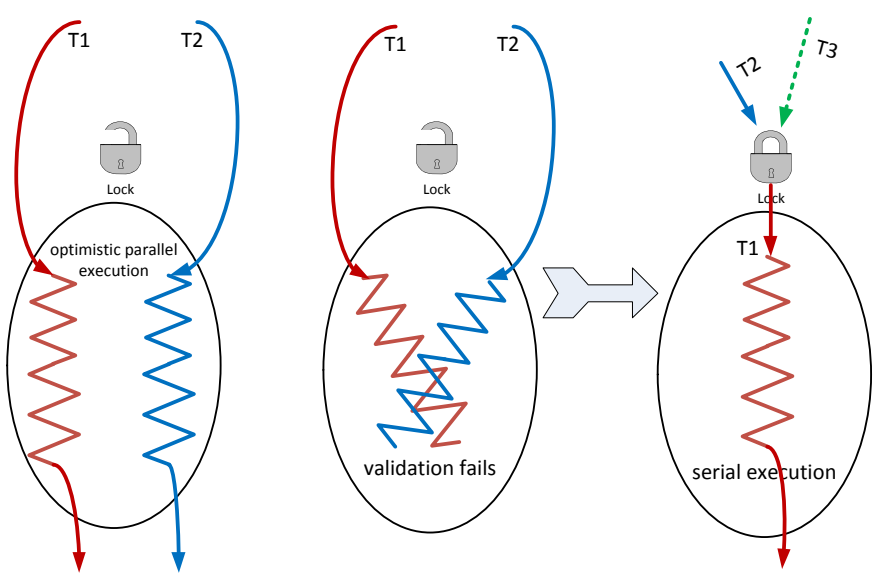

Figure 3. Lock elision (left), conflict (middle), and serial execution (right)

that Haswell was not the first CPU with hardware support for transactional memory, for example IBM's Blue Gene/Q supercomputers [13] and System z mainframes [14] offered it before, but it is the first mainstream CPU to implement HTM. And in hardware, transactional memory can be implemented much more efficiently than in software: Haswell uses its highly optimized cache coherence protocol, which is needed for all multi-core processors anyway, to track read and write set collisions [15]. Therefore, Haswell offers HTM nearly for free.

Even though HTM is very efficient, there are also some restrictions. First of all, the size of a hardware transaction is limited. For the Haswell architecture it is limited to the size of the $\mathrm{L} 1$ cache, which is $32 \mathrm{~KB}$. This implies that, in general, it is not possible to simply execute a database transaction as one monolithic HTM transaction. Even mediumsized database transactions would be too large. Second, in the case of conflicts, the transaction fails. In this case the CPU undoes all changes, and then reports an error that the application has to handle. And finally, a transaction might fail due to spurious implementation details like cache associativity limits, certain interrupts, etc. So, even though in most cases HTM will work fine, there is no guarantee that a transaction will ever succeed (if executed as an HTM transaction).

Therefore, Intel proposes (and explicitly supports by specific instructions) using transactional memory for lock elision [15]. Conceptually, this results in code like the following:

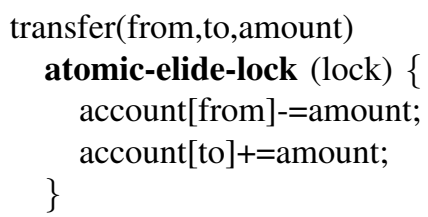

Here, we still have a lock, but ideally the lock is not used at all-it is elided. When the code is executed, the CPU starts an HTM transaction, but does not acquire the lock as shown on the left-hand side of Figure 3. Only when there is a conflict the transaction rolls back, acquires the lock, and is then executed non-transactionally. The right-hand side of Figure 3 shows the fallback mechanism to exclusive serial execution, which is controlled via the (previously elided) lock. This lock elision

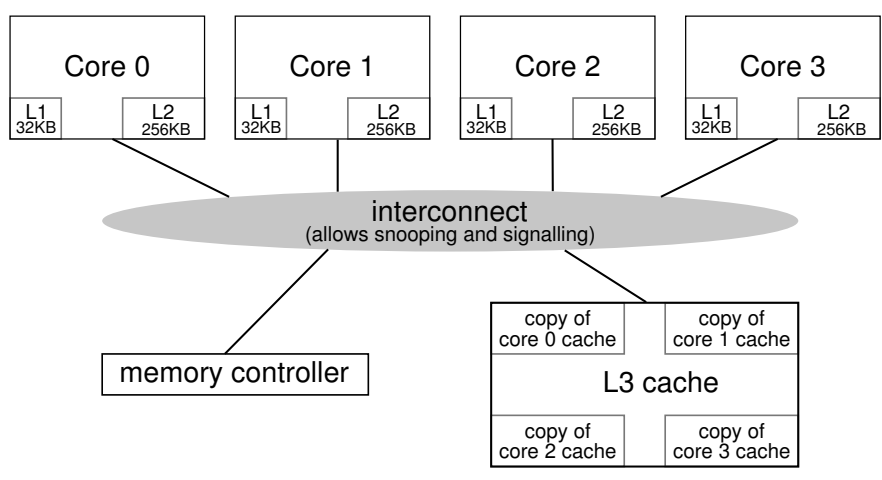

Figure 4. Intel cache architecture

mechanism has two effects: 1) ideally, locks are never acquired and transactions are executed concurrently as much as possible 2 ) if there is an abort due to a conflict or hardware-limitation, there is a "slow path" available that is guaranteed to succeed.

\subsection{Caches and Cache Coherency}

Even though Intel generally does not publish internal implementation details, Intel did specify two important facts about Haswell's HTM feature [15]:

- The cache coherency protocol is used to detect transactional conflicts.

- The L1 cache serves as a transactional buffer.

Therefore, it is crucial to understand Intel's cache architecture and coherency protocol.

Because of the divergence of DRAM and CPU speed, modern CPUs have multiple caches in order to accelerate memory accesses. Intel's cache architecture is shown in Figure 4, and consists of a local L1 cache ( $32 \mathrm{~KB})$, a local L2 cache $(256 \mathrm{~KB})$, and a shared L3 cache (2-45 MB). All caches use 64 byte cache blocks (lines) and all caches are transparent, i.e., programs have the illusion of having only one large main memory. Because on multi-core CPUs each core generally has at least one local cache, a cache coherency protocol is required to maintain this illusion.

Both Intel and AMD use extensions of the well-known MESI protocol [16]. The name of the protocol derives from the four states that each cache line can be in (Modified, Exclusive, Shared, or Invalid). To keep multiple caches coherent, the caches have means of intercepting ("snooping") each other's load and store requests. For example, if a core writes to a cache line which is stored in multiple caches (Shared state), the state must change to Modified in the local cache and all copies in remote caches must be invalidated (Invalid state). This logic is implemented in hardware using the cache controller of the shared L3 cache that acts as a central component where all coherency traffic and all DRAM requests pass through.

The key insight that allows for an efficient HTM implementation is that the L1 cache can be used as a local buffer. All transactionally read or written cache lines are marked and the propagation of changes to other caches or main memory is prevented until the transaction commits. Read/write and write/write conflicts are detected by using the same snooping logic that is used to keep the caches coherent. And since the 


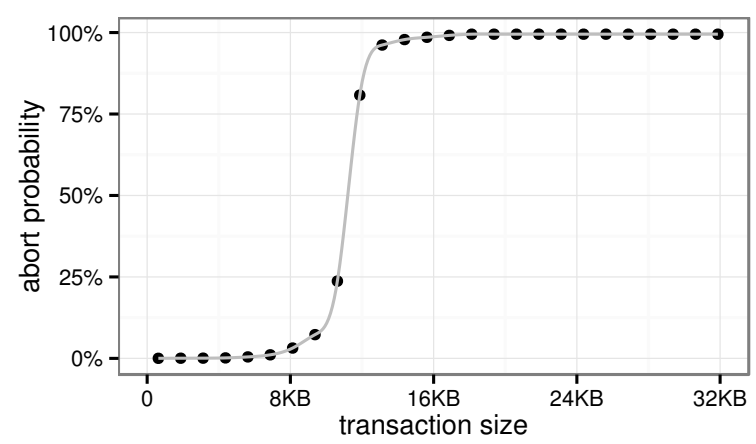

Figure 5. Aborts from random memory writes

MESI protocol is always active and commits/aborts require no inter-core coordination, transactional execution on Haswell CPUs incurs almost no overhead. The drawback is that the transaction size is limited to the $\mathrm{L} 1$ cache. This is fundamentally different from IBM's Blue Gene/Q architecture, which allows for up to $20 \mathrm{MB}$ per transaction using a multi-versioned L2 cache, but has relatively large runtime overhead [13].

Besides the nominal size of the $\mathrm{L} 1$ cache, another limiting factor for the maximum transaction size is cache associativity. Caches are segmented into sets of cache lines in order to speed up lookup and to allow for an efficient implementation of the pseudo-LRU replacement strategy (in hardware). Haswell's L1 cache is 8-way associative, i.e., each cache set has 8 entries. This has direct consequences for HTM, because all transactionally read or written cache lines must be marked and kept in the L1 cache until commit or abort. Therefore, when a transaction writes to 9 cache lines that happen to reside in the same cache set, the transaction is aborted. And since the mapping from memory address to cache set is deterministic (bits 7-12 of the address are used), restarting the transaction does not help, and an alternative fallback path is necessary for forward progress.

In practice, bits 7-12 of memory addresses are fairly random, and aborts of very small transactions are unlikely. Nevertheless, Figure 5 shows that the abort probability quickly rises when more than 128 random cache lines (only about one quarter of the L1 cache) are accessed ${ }^{1}$. This surprising fact is caused by a statistical phenomenon related to the birthday paradox: For example with a transaction size of $16 \mathrm{~KB}$, for any one cache set it is quite unlikely that it contains more than 8 entries. However, at the same time, it is likely that at least one cache set exceeds this limit. An eviction of a line from the cache automatically leads to a failure of this transaction as it would become impossible to detect conflicting writes to this cache line.

The previous experiment was performed with accesses to memory addresses fully covered by the translation lookaside buffer (TLB). TLB misses do not immediately cause transactions to abort, because, on x86 CPUs, the page table lookup is performed by the hardware (and not the operating system). However, TLB misses do increase the abort probability, as they

1. The experiments in this section were performed on an Intel i5 4670T.

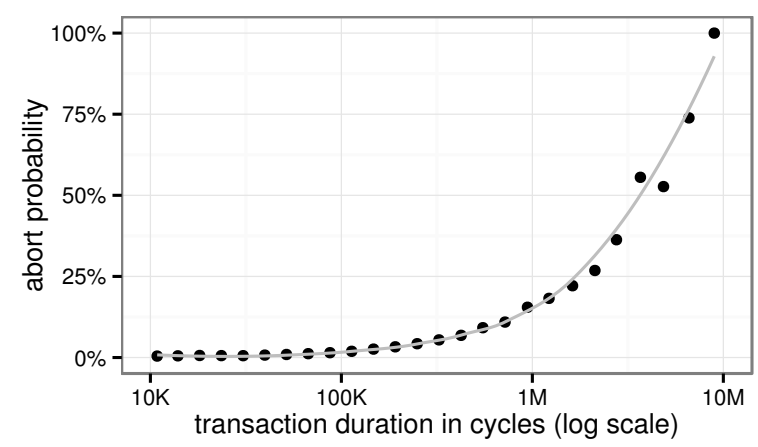

Figure 6. Aborts from transaction duration

cause additional memory accesses during page table walks.

Besides memory accesses, another important reason for transactional aborts is interrupts. Such events are unavoidable in practice and limit the maximum duration of transactions. Figure 6 shows that transactions that take more than 1 million CPU cycles (about $0.3 \mathrm{~ms}$ ) will likely be aborted, even if they only compute and execute no memory operations. These results clearly show that Haswell's HTM implementation cannot be used for long-running transactions but is designed for short critical sections. Despite these limitations we found that Haswell's HTM implementation offers excellent scalability as long as transactions are short and free of conflicts with other transactions.

\section{Synchronization on MANY-CORE CPUS}

Intel's current medium-level server platform Haswell EP has up to 18 cores per socket. Commodity servers, which often have two sockets, will soon commonly have over 50 hardware threads, so executing transactions using only a single thread would waste most of this computational power. To execute transactional workloads, a DBMS must therefore provide (1) high-level concurrency control to logically isolate transactions, and (2) a low-level synchronization mechanism to prevent concurrent threads from corrupting internal data structures. Both aspects are very important, as any of them may prevent scalability. Our concurrency control scheme is described in Section 4. In this section, we instead focus on the low-level synchronization aspect. We experimentally evaluate HTM on a Haswell system with 28 cores and compare it with common synchronization alternatives like latching.

For our experiments we use a Haswell EP system with two Intel E5-2697 v3 processors that are connected through QPI. The processor is depicted in Figure 7 and has 14 cores, i.e., in total, the system has 28 cores (56 HyperThreads). The figure also shows that the CPU has two internal communication rings that connect cores and $2.5 \mathrm{MB}$ slices of the L3 cache. An internal link connects these two rings, but is not to be confused with the QPI interconnect that connects the two sockets of the system. The system supports two modes, which can be selected in the systems' BIOS:

- The hardware can hide the internal ring internal structure, i.e., our two-socket system would expose two NUMA nodes with 14 cores each. 


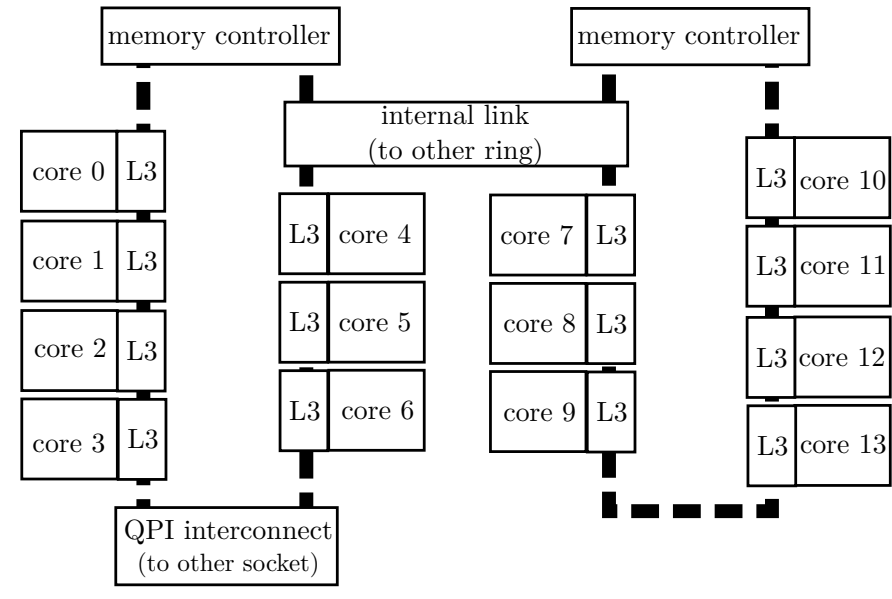

Figure 7. Intel E5-2697 v3

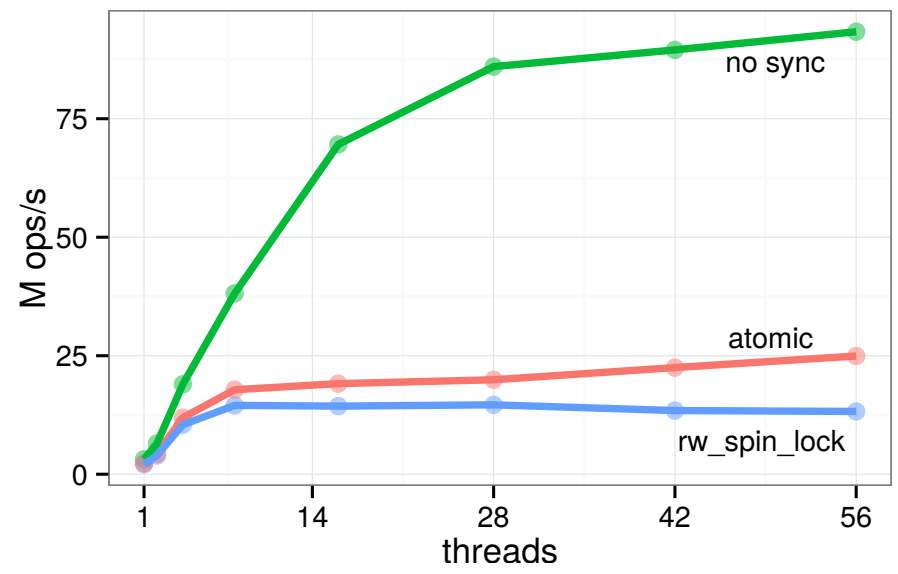

Figure 8. Lookups in a search tree with $64 \mathrm{M}$ entries

- In the "cluster on die" configuration each internal ring is exposed as a separate NUMA node, i.e., our two-socket system has four NUMA nodes with 7 cores each.

Using the first setting, each socket has $35 \mathrm{MB}$ of L3 cache but higher latency, because an L3 access often needs to use the internal link. The cluster-on-die configuration, which we use for all experiments, has lower latency and 17.5MB of cache per "cluster", each of which consists of 7 threads.

As has been widely reported, all Haswell systems shipped so far, including both our test systems, contain a hardware bug in the Transactional Synchronization Extensions (TSX). According to Intel, this bug occurs "under a complex set of internal timing conditions and system events". We have not encountered this bug during our tests. It seems to be very rare, as evidenced by the fact that it took Intel over a year to even find it. Furthermore, Intel has announced that the bug will be fixed in upcoming CPU generations.

\subsection{The Perils of Latching}

Traditionally, database systems synchronize concurrent access to internal data structures (e.g., index structures) with latches. Internally, a latch is implemented using atomic operations like compare-and-swap, which allow to exclude other threads from entering a critical section. To increase concurrency, read/write latches are often used, which, at any time, allow multiple concurrent readers but only a single writer. Unfortunately, latches do not scale on modern hardware as Figure 8, which performs lookups in an Adaptive Radix Tree [17], shows. The curve labeled as "rw_spin_lock" shows the performance when we add a single read/write latch at the root node of the tree ${ }^{2}$. With many cores, using no synchronization is faster by an order of magnitude! Note that this happens on a read-only workload without any logical contention, and is not caused by a bad latch implementation ${ }^{3}$ : When we replace the latch with a single atomic integer increment operation, which is the cheapest possible atomic write operation, the scalability is almost as bad as with the latch.

The reason for this behavior is that to acquire a latch, CPUs must acquire exclusive access to the cache line where the latch is stored. As a result, threads compete for this cache line, and every time the latch is acquired, all copies of this cache line are invalidated in all other cores ("cache line ping-pong"). This happens even with atomic operations like atomic increment, although this operation never fails in contrast to compare-andswap, which is usually used to implement latches. Note that latches also result in some overhead during single-threaded execution, but this overhead is much lower as the latch cache line is not continuously invalidated. Cache line ping-pong is often the underlying problem that prevents systems from scaling on modern multi-core CPUs.

\subsection{Latch-Free Data Structures}

As a reaction to the bad scalability of latching some systems use latch-free data structures. Microsoft's in-memory transaction engine Hekaton, for example, uses a lock-free hash table and the latch-free Bw-Tree [18] as index structures. In the latch-free approach read accesses can proceed in a nonblocking fashion without acquiring any latches and without waiting. Writes must make sure that any modification is performed using a sequence of atomic operations while ensuring that simultaneous reads are not disturbed. Since readers do not perform writes to global memory locations, this approach generally results in very good scalability for workloads that mostly consist of reads. However, latch-free data structure have a number of disadvantages:

- The main difficulty is that, until the availability of Hardware Transactional Memory, CPUs provided only very primitive atomic operations like compare-and-swap, and a handful of integer operations. Synchronizing a non-trivial data structure with this limited tool set is very difficult and bug-prone, and for many efficient data structures, including the Adaptive Radix Tree, so far, no latchfree synchronization protocol exists. In practice, data structures must be designed with latch-freedom in mind, and the available atomic operations restrict the design space considerably.

2. In reality, one would use lock-coupling and one latch at each node, so the performance would be even worse.

3. We used spin_rw_mutex from the Intel Thread Building Blocks library. 


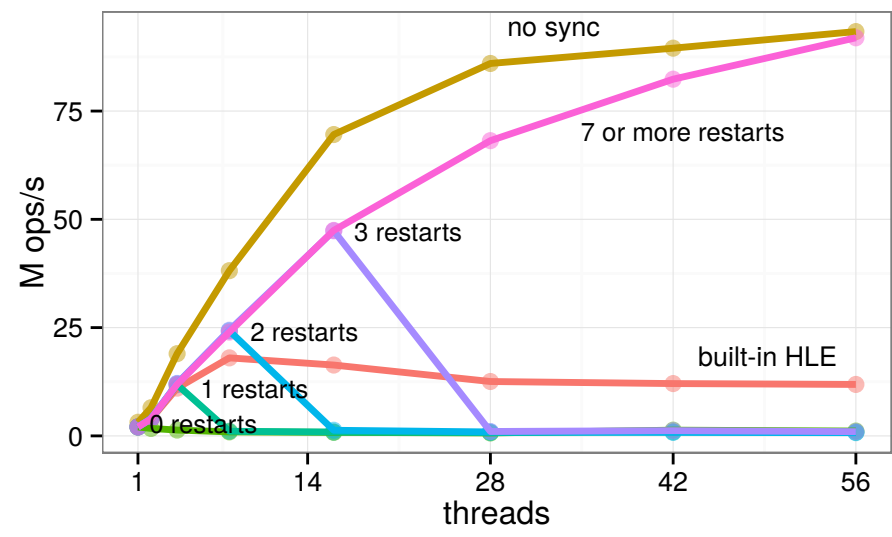

Figure 9. Lookups in a search tree with 64M entries using a varying number of HTM restarts

- And even if one succeeds in designing a latch-free data structure, this may not guarantee optimal performance. The reason is that usually additional indirections must be introduced, which often add significant overhead in comparison with an unsynchronized variant of the data structure. The Bw-tree [18], for example, requires a page table indirection, which must be used on each node access and incurs additional cache misses.

- Finally, memory reclamation is an additional problem, because a thread can never be sure when it is safe to reclaim memory, because concurrent readers might still be active. An additional mechanism (e.g., epochbased reclamation), which again adds some overhead, is required to allow for safe memory reclamation.

For these reasons, and because database systems internally use many different custom data structures, we find latchfreedom too difficult in practice. Hardware Transactional Memory offers an easy to use, and, as we will show, efficient alternative. In particular, HTM has no memory reclamation issues and one can simply wrap each data structure access in a hardware transaction. This means that data structures can be designed without spending too much thought on how to synchronize them-though some understanding of how HTM works and its limitations is certainly beneficial.

\subsection{Hardware Transactional Memory on Many-Core Systems}

Figure 9 shows the performance of HTM on the same readonly workload as Figure 8. We compare different lock elision approaches (with a single global, elided latch), and we again show the performance without synchronization as a theoretical upper bound. Surprisingly, the built-in hardware lock elision (HLE) instructions do not scale well when more than 4 cores are used. The internal implementation of HLE is not disclosed, but the reason for its bad performance is likely an insufficient number of restarts. As we have mentioned previously, a transaction can abort spuriously for many reasons, thus a transaction should retry a number of times instead of giving up immediately and acquiring the fallback latch. The graph shows that for a read-only workload, restarting at least 7 times is necessary, though a higher number of restarts also works fine.

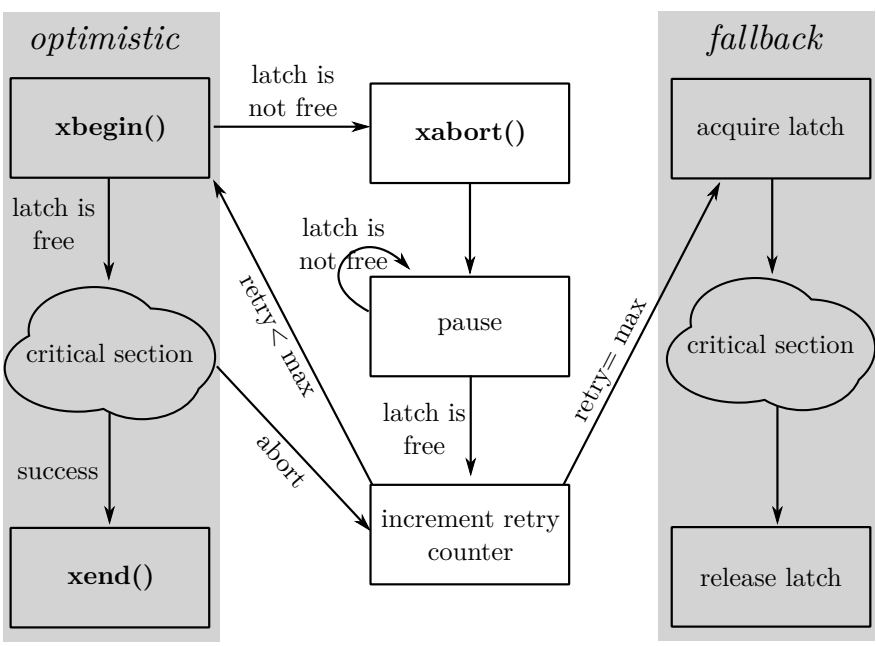

Figure 10. Implementation of lock elision with restarts using RTM operations

Therefore, we implemented lock elision manually using the restricted transactional memory (RTM) primitives xbegin, xend, xabort, but with a configurable number of restarts. Figure 10 shows the state diagram of our implementation. Initially the optimistic path (left-hand side of the diagram) is taken, i.e., the critical section is simply wrapped by xbegin and xend instructions. If an abort happens in the critical section (e.g, due to a read/write conflict), the transaction is restarted a number of times before falling back to actual latch acquisition (right-hand side of the diagram). Furthermore, transactions add the latch to their read set and only proceed into the critical section optimistically when the latch is free. When it is not free, this means that another thread is in the critical section exclusively (e.g., due to a code that cannot succeed transactionally). In this case, the transaction has to wait for the latch to become free, but can then continue to proceed optimistically using RTM. Note that all this logic is completely hidden behind a typical acquire/release latch interface, and - once it has been implemented-it can be used just as easily as ordinary latches or HLE. Furthermore, as Diegues and Romano [19] have shown, the configuration parameters of the restart strategy can be determined dynamically.

When sufficient restarts are used, the overhead of HTM in comparison to unsynchronized access is quite low. Furthermore, we found that HyperThreading improves performance for many workloads, though one has to keep in mind that because each pair of HyperThreads shares one L1 cache, the effective maximum working set size is halved, so there might be workloads where it is beneficial to avoid this feature.

Good scalability on read-only workloads should be expected, because only infrequent, transient failures occur, thus we now turn our attention to more challenging workloads. Figure 11 reports the results for a random insert workload with 30 restarts and different memory allocators. The default glibc memory allocator completely prevents scalability. The tcmalloc ${ }^{4}$ (Thread-Caching Malloc) allocator improves perfor-

\footnotetext{
4. http://goog-perftools.sourceforge.net/doc/tcmalloc.html
} 


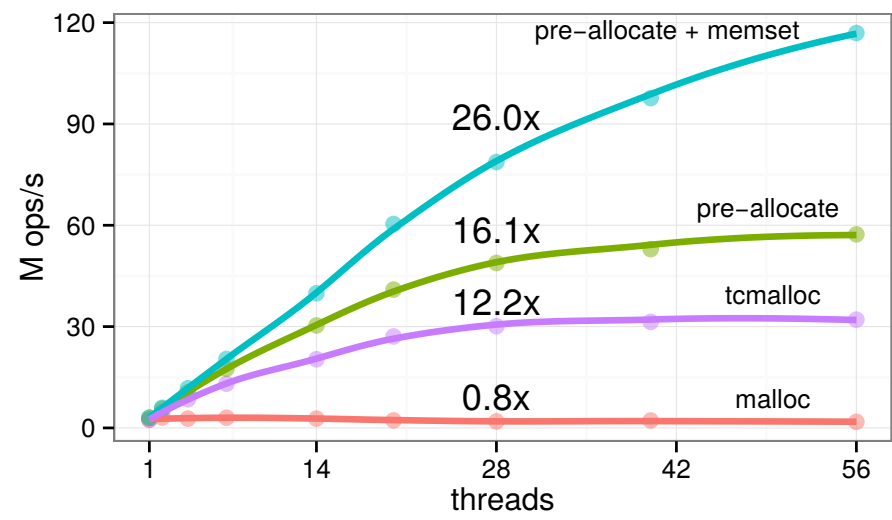

Figure 11. 64M random inserts using different memory allocators and 30 restarts

mance considerably, but the speedup with 28 threads is still only $12.2 \times$. Optimal scalability is only achieved with preallocated and initialized memory, which results in a speedup of $26.0 \times$ with 28 cores. Initialization is important, because the first write to a freshly-allocated memory page always causes the transaction to abort as an operating system trap that initializes the page happens. Although these page initialization operations are quite infrequent (less than $5 \%$ of the singlethreaded execution time), they cause the elided latch to be acquired and therefore full serialization of all threads occurs.

So far, in all experiments the memory was interleaved between the four memory nodes. In order to investigate the NUMA effects on HTM, we repeated the insert and lookup experiments with 1 and 7 threads, but forced the threads and memory allocations to one cluster, one socket, or two sockets:

\begin{tabular}{l|r|r|r} 
& 1 cluster & 1 socket & 2 sockets \\
\hline insert (1 thread) & 5.3 & 4.3 & 3.0 \\
insert (7 threads) & 30.6 & 26.8 & 20.2 \\
lookup (1 thread) & 9.2 & 5.4 & 3.6 \\
lookup (7 threads) & 53.0 & 36.0 & 24.5
\end{tabular}

The results are in $\mathrm{M}$ ops/s and show that there are significant NUMA effects: remote accesses are up to a factor 2.5 more expensive than local accesses. At the same time, HTM scales very well even across clusters or sockets. To some extent, this is not very surprising, because NUMA systems have an effective cache coherency protocol implementation, which is also used for efficient transactional conflict detection.

Finally, let us close with an experiment that shows that modern CPUs do not scale under very high contention regardless which synchronization primitives are used. Figure 12 shows the performance for an extreme workload where there is a single atomic counter that is incremented by all threads. In all cases performance degrades with more cores due to cache line ping-pong. For workloads with high contention one needs an approach that solves the root cause, physical contention, e.g., by duplicating the contended item [20].

\subsection{Discussion}

Figure 13 schematically compares the scalability and the ease of use (for the database system programmer) of latching, lockfree data structures, and HTM. The great advantage of HTM

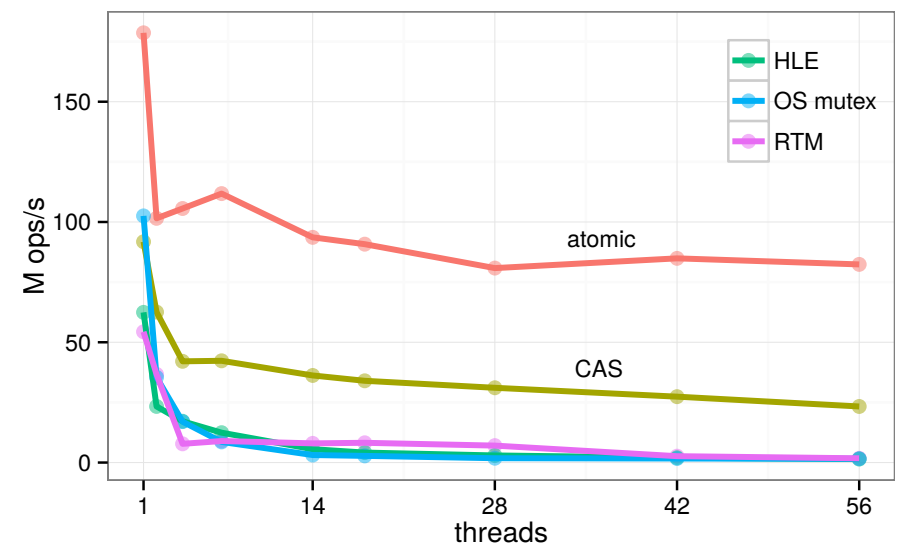

Figure 12. Incrementing a single, global counter (extreme contention)

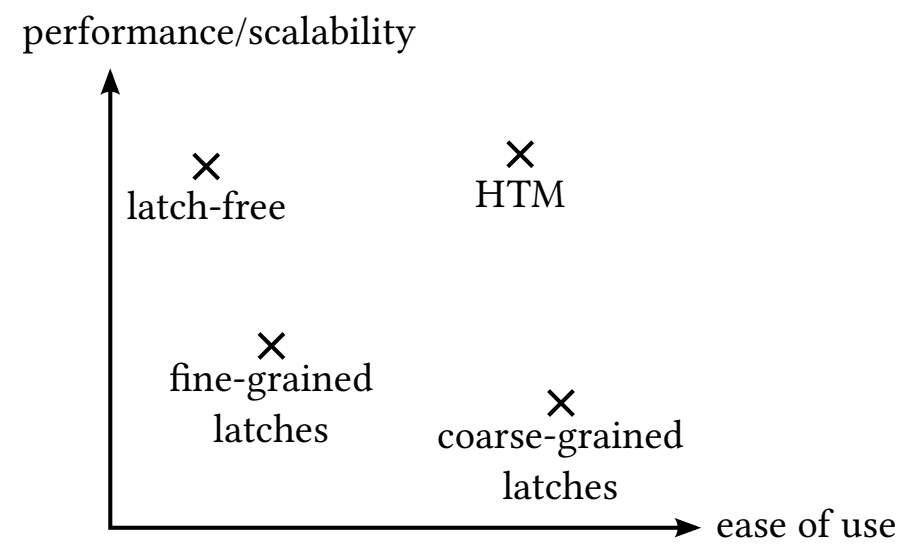

Figure 13. Schematic comparison of different synchronization approaches

is that it is the only synchronization approach that offers good performance and scalability while being easy to use. Our experiments with hardware transactional memory on a 28-core system with Non-Uniform Memory Access (NUMA) have shown that HTM can indeed scale to large systems. However, we have also seen that to get good performance a number of important points must be considered:

- The built-in Hardware Lock Elision feature does not scale when many cores are used.

- Instead, one should implement lock elision using the Restricted Transactional Memory primitives, and set the number of retries to 20 or more.

- Additionally, one has to make sure that the percentage of transactions that cannot be executed transactionally, e.g., due to kernel traps, is very low. Otherwise failed lock elision will cause serialization, and Amdahl's Law severely limits scalability.

- HTM, just like latching or atomic operations, does not scale under very high contention workloads.

Nevertheless, HTM is a powerful and efficient new synchronization primitive for many-core systems. 


\section{HTM-supported Transaction Manage- MENT}

As databases are expected to offer ACID transactions, they have to implement a mechanism to synchronize concurrent transactions. The traditional concurrency control method used in most database systems is some variant of two-phase locking (2PL). Before accessing a database item (tuple, page, etc.), the transaction acquires a lock in the appropriate lock mode (shared, exclusive, etc.). Conflicting operations, i.e., conflicting lock requests, implicitly order transactions relative to each other and thus ensure serializability.

In the past this model worked very well. Concurrent transaction execution was necessary to hide I/O latency, and the costs for checking locks was negligible compared to the processing costs in disk-based systems. However, this has changed in modern systems, where large parts of the data are kept in main memory, and where query processing is increasingly CPU bound. In such a setup, lock-based synchronization constitutes a significant fraction of the total execution time, in some cases even dominates the processing [7], [21].

In particular, it is very hard to decide at which granularity latching/locking should be performed: if very finegrained latching is used, the additional overhead will annihilate any speedup from parallelism; with coarse-grained latches, parallelism is, limited. For non-trivial programs, this is a very difficult problem, and the most efficient choice can often only be decided empirically. The granularity problem is even more difficult for a database system because it must efficiently support arbitrary workloads. With hardware support, transactional memory offers an elegant solution: As long as conflicts are infrequent, HTM offers the parallelism of finegrained latching, but without its overhead; if hotspots occur frequently, the best method in main-memory databases is serial execution, which is exactly the fallback path for HTM conflicts. Therefore, HTM is a highly promising building block for high performance database systems.

\subsection{Mapping Database Transactions to HTM Trans- actions}

As the maximum size of hardware transactions is limited, only a database transaction that is small can directly be mapped to a single hardware transaction. Therefore, we assemble complex database transactions by using hardware transactions as building blocks, as shown in Figure 14. The key idea here is to use a customized variant of timestamp ordering (TSO) to "glue" together these small hardware transactions. TSO is a classic concurrency control technique, which was extensively studied in the context of disk-based and distributed database systems [22], [23]. For disk-based systems, TSO is not competitive to locking because most read accesses result in an update of the read timestamp, and thus a write to disk. These timestamp updates are obviously much cheaper in RAM. On the opposite, fine-grained locking is much more expensive than maintaining timestamps in main memory, as we will show in Section 6.

Timestamp ordering uses read and write timestamps to identify read/write and write/write conflicts. Each transaction

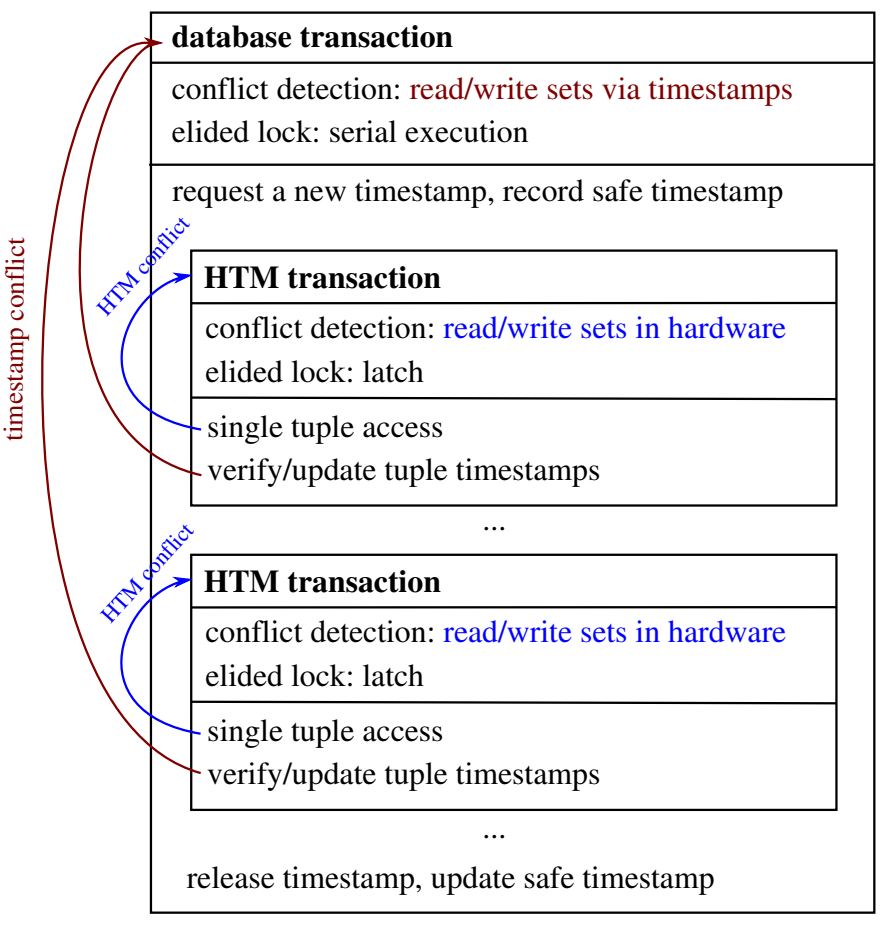

Figure 14. Transforming database transactions into HTM transactions

is associated with a monotonically increasing timestamp, and whenever a data item is read or updated its associated timestamp is updated, too. The read timestamp of a data item records the youngest reader of this particular item, and the write timestamp records the last writer. This way, a transaction recognizes if its operation collides with an operation of a "younger" transactions (i.e., a transaction with a larger timestamp), which would be a violation of transaction isolation. In particular, an operation fails if a transaction tries to read data from a younger transaction, or if a transaction tries to update a data item that has already been read by a younger transaction. Note that basic TSO [22] has to be refined to prevent phantoms. Furthermore, some care is needed to prevent non-recoverable schedules, as by default transactions are allowed to read data from older, but potentially noncommitted, transactions.

To resolve both issues (phantoms and dirty reads), we deviate from basic TSO by introducing a "safe timestamp", i.e., a point in time where it is known that all older transactions have already been committed. With classical TSO, when a transaction tries to read a dirty data item (marked by a dirty bit) from another transaction, it must wait for that transaction to finish. In main-memory database systems running at full clock speed, waiting is very undesirable.

We avoid both waiting and phantom problems with the safe timestamp concept. The safe timestamp $\mathrm{TS}_{\text {safe }}$ is the youngest timestamp for which holds: All transactions with an older timestamp $\mathrm{TS}_{\text {old }}$ with old $\leq$ safe have already been committed or aborted. While regular TSO compares transaction timestamps directly, we compare timestamps to the safe timestamp of each transaction: Everything that is older than the safe timestamp can be safely read, and everything that has been 
read only by transactions up to the safe timestamp can safely be modified. Note that we could also access or modify some tuples with newer timestamps, namely those from transactions that have already committed in between this transaction's begin and now. But this would require complex and expensive checks during tuple access, in particular if one also wants to prevent phantoms. We therefore use the safe timestamp as a cheap, though somewhat conservative, mechanism to ensure serializability. In the scenario

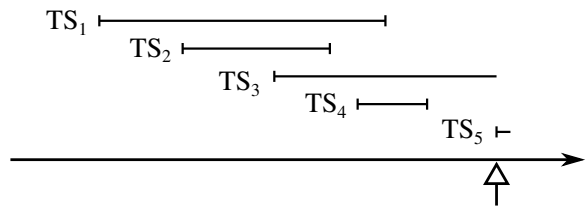

the safe timestamp of $\mathrm{TS}_{5}$ would be set to $\mathrm{TS}_{2}$. So transaction $\mathrm{TS}_{5}$ would validate its read access such that only data items with a write timestamp $\mathrm{TS}_{\mathrm{W}} \leq \mathrm{TS}_{2}$ are allowed. Write accesses on behalf of $\mathrm{TS}_{5}$ must additionally verify that the read timestamp of all items to be written satisfies the condition $\mathrm{TS}_{\mathrm{R}} \leq \mathrm{TS}_{2}$. Obviously, a read or write timestamp $\mathrm{TS}=\mathrm{TS}_{5}$ is permitted as well-in case a transaction accesses the same data item multiple times.

\subsection{Conflict Detection and Resolution}

In our scheme, the read and the write timestamps are stored at each tuple. After looking up a tuple in an index, its timestamp(s) must be verified and updated. Each single tuple access, including index traversal and timestamp update, is executed as a hardware transaction using lock elision. The small granularity ensures that false aborts due to hardware limitations are very unlikely, because Haswell's hardware transactions can access dozens of cache lines (cf. Section 2).

Nevertheless, two types of conflicts may occur: If HTM detects a conflict (short blue arrows in Figure 14), the hardware transaction is restarted, but this time the latch is acquired. Rolling back a hardware transaction is very cheap, as it only involves invalidating the transactionally modified cache lines, and copies of the original content can still be found in the L2 and/or L3 cache.

For timestamp conflicts, which are detected in software (long red arrows in Figure 14), the system must first roll back the database transaction. This rollback utilizes the "normal" logging and recovery infrastructure of the database system, i.e., the undo-log records of the partial database transaction are applied in an ARIES-style compensation [24]. Then, the transaction is executed serially by using a global lock, rolling the log forward again. This requires logical logging and non-interactive transactions, as we cannot roll a user action backward or forward. We use snapshots to isolate interactive transactions from the rest of the system [21]. The fallback to serial execution ensures forward progress, because in serial execution a transaction will never fail due to conflicts. Note that it is often beneficial to optimistically restart the transaction a number of times instead of resorting to serial execution immediately, as serial execution is very pessimistic and prevents parallelism.
Figure 15 details the implementation of a database transaction using lock elision and timestamps. The splitting of stored procedures into smaller HTM transactions is fully automatic (done by our compiler) and transparent for the programmer. As shown the example, queries or updates (within a database transaction) that access a single tuple through a unique index are directly translated into a single HTM transaction. Larger statements like non-unique index lookups should be split into multiple HTM transactions, e.g., one for each accessed tuple. The index lookup and timestamp checks are protected using an elided latch, which avoids latching the index structures themselves. The implementation of the HTM latch is described in Section 3.3.

\subsection{Optimizations}

How the transaction manager handles timestamp conflicts is very important for performance. If the conflict is only caused by the conservatism of the safe timestamp (i.e., regular TSO would have no conflict), it is sometimes possible to avoid rolling back the transaction. If the conflicting transaction has a smaller timestamp and has already finished, the apparent conflict can be ignored. This optimization is possible because the safe timestamp cannot overtake a currently running transaction's timestamp.

As mentioned before, it is often beneficial to restart an aborted transaction a number of times, instead of immediately falling back to serial execution. In order for the restart to succeed, the safe timestamp must have advanced past the conflict timestamp. Since this timestamp is available (it triggered the abort), the transaction can wait, while periodically recomputing the safe timestamp until it has advanced sufficiently. Then the transaction can be restarted with a new timestamp and safe timestamp. The disadvantage of this approach is that during this waiting period no useful work is performed by the thread.

A more effective strategy is to suspend the aborted transaction and execute other transactions instead. Once the safe timestamp has advanced past the conflicting transaction's timestamp that transaction can be resumed. This strategy avoids wasteful waiting. We found rollback and re-execution to be quite cheap because the accessed data is often in cache. Therefore, our implementation immediately performs an abort after a timestamp conflict, as shown in Figure 15, and executes other transactions instead, until the safe timestamp has sufficiently advanced. We additionally limit the number of times a transaction is restarted before falling back to serial execution-thus ensuring forward progress.

While our description here and also our initial implementation uses both read and write timestamps, it is possible to avoid read timestamps. Read timestamps are a bit unfortunate, as they can cause "false" HTM conflicts due to parallel timestamp updates, even though the read operations themselves would not conflict. Semantically the read timestamps are used to detect if a tuple has already been read by a newer transaction, which prohibits updates by older transactions (as they would destroy serializability). However, the read timestamps can be avoided by keeping track of the write timestamps of all data items accessed (read or written) by a certain transaction. 


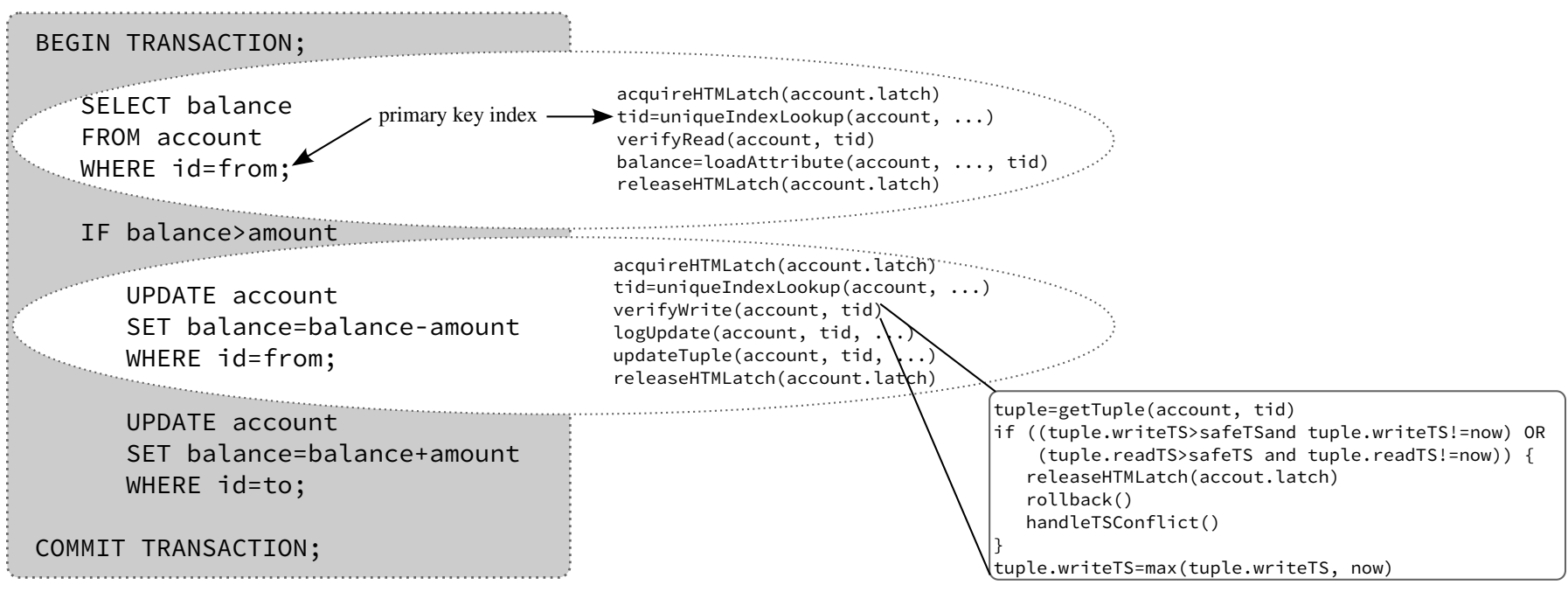

Figure 15. Implementing database transactions with timestamps and lock elision

Then, at commit time, the transaction re-examines the write timestamps of all data items and aborts if any one of them has changed [25], ensuring serializability. We plan to implement this technique in future work, and expect to get even better performance in the case of read hotspots.

It is illustrative to compare our scheme to software transactional memory (STM) systems. Indeed, our scheme can be considered an HTM-supported implementation of STM. However, we get significantly better performance than pure STM by exploiting DBMS domain knowledge. For example, index structures are protected from concurrent modifications by the HTM transaction, but are not tracked with timestamps, as full transaction isolation would in fact be undesirable there. This is similar to B-tree latching in disk-based systemshowever, at minimal cost. The indexed tuples themselves are isolated via timestamps to ensure serializable transaction behavior. Note further that our interpretation of timestamps is different from regular TSO [22]: Instead of deciding about transaction success and failure as in TSO, we use timestamps to detect intersecting read/write sets, just like the hardware itself for the HTM part. In the case of conflicts, we do not abort the transaction or retry with a new timestamp an indefinite number of times, but fall back to the more restrictive sequential execution mode that ensures forward progress and guarantees the eventual success of every transaction.

\section{HTM-Friendly Data Storage}

Transactional memory synchronizes concurrent accesses by tracking read and write sets. This avoids the need for finegrained locking and greatly improves concurrency as long as objects at different memory addresses are accessed. However, because HTM usually tracks accesses at cache line granularity, false conflicts may occur. For example, if the two data items A and B happen to be stored in a single cache line, a write to A causes a conflict with B. This conflict would not have occurred if each data item had its own dedicated lock. Therefore, HTM presents additional challenges for database systems that must be tackled in order to efficiently utilize this feature.
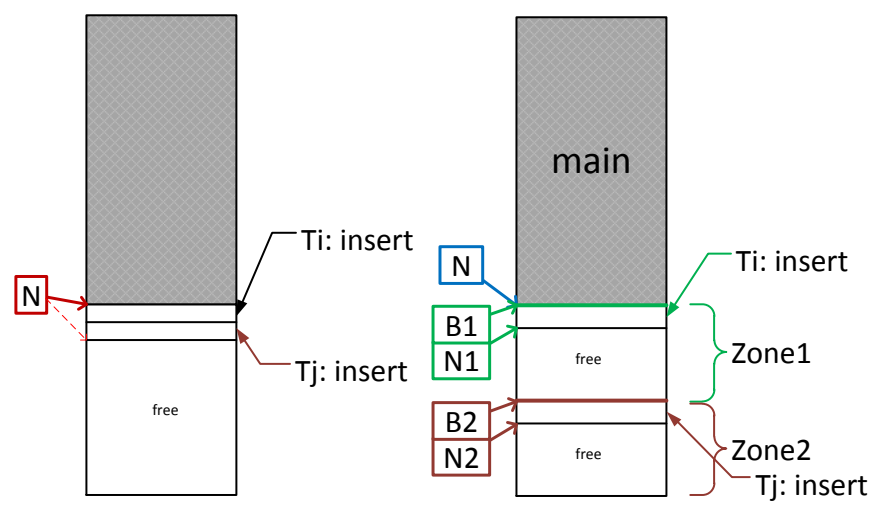

Figure 16. Avoiding hotspots by zone segmentation

\subsection{Data Storage with Zone Segmentation}

With a straightforward contiguous main-memory data layout, which is illustrated on the left-hand side of Figure 16, an insert into a relation results in appending the tuple to the end of the relation. It is clear that such a layout does not allow concurrent insertions, because each insert writes to the end of the relation. Additionally, all inserts will try to increment some variable $N$ which counts the number of tuples. The memory location at the end of the table and the counter $N$ are hotspots causing concurrent inserts to fail.

In order to allow for concurrent inserts, we use multiple zones per relation, as shown on the right-hand side of Figure 16. Each relation has a constant number of these zones, e.g., two times the number of hardware threads. A random zone number is assigned to each transaction, and all inserts of that transaction use this local zone. The same zone number is also used for inserts into other relations. Therefore, with an appropriately chosen number of zones, concurrent inserts can proceed with only a small conflict probability, even if many relations are affected. Besides the relatively small insert zones, each relation has a main zone where, for large relations, most tuples are stored.

The boundary is stored in a counter $N$. For each zone $i$, the 


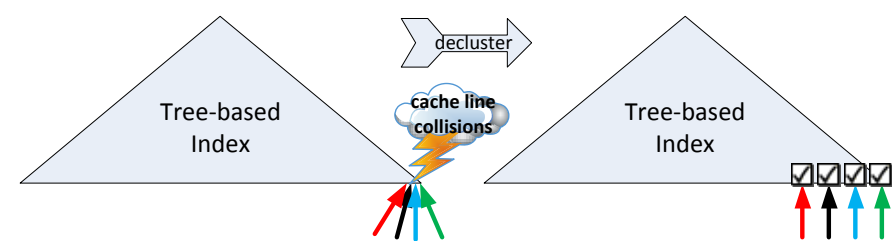

Figure 17. Declustering surrogate key generation

base $B_{i}$ and the next insert position $N_{i}$ are maintained. When a zone becomes full (i.e., when $N_{i}$ reaches $B_{i+1}$ ), it is collapsed into the neighboring zone, and a new zone at the end of the table is created. Note that no tuples are copied and the tuple identifiers do not change, only the sizes of zones need to be adjusted. As a consequence, collapsing zones does not affect concurrent access to the tuples. Eventually, the insert zones are collapsed with the large contiguous main area. For a mainmemory databases this guarantees very fast scan performance at clock speed during query processing. The counters $N_{i}$ and $B_{i}$ should be stored in separate cache lines for each zone, as otherwise unnecessary conflicts occur while updating these values.

\subsection{Index Structures}

Besides the logical isolation of transactions using 2PL or TSO, database systems must isolate concurrent accesses to index structures. In principle, any data structure can be synchronized using HTM by simply wrapping each access in a transaction. In this section we first discuss how scalability can be improved by avoiding some common types of conflicts, before showing that HTM has much better performance than traditional index synchronization via fine-grained latches.

One common problem is that indexes often have a counter that stores the number of key/value pairs and prevents concurrent modifications. Fortunately, this counter is often not needed and can be removed. For data structures that allocate small memory chunks, another source of HTM conflicts is memory allocation. This problem can be solved by using an allocator that has a thread-local buffer.

Surrogate primary keys are usually implemented as ascending integer sequences. For tree-based index structures, which maintain their data in sorted order, this causes HTM conflicts because all concurrent inserts try to access memory locations in the same vicinity, as illustrated on the left-hand side of Figure 17. This problem is very similar to the problem of concurrently inserting values into a table discussed above, and indeed the solution is similar: If permitted by the application, the integer sequence is partitioned into multiple constant-sized chunks and values are handed out from one of the chunks depending on the transactions' zone number. This prevents interference of parallel index tree insertions as they are spread across different memory locations - as shown on the right of Figure 17. Once all values from a chunk are exhausted, the next set of integers is assigned to it. Note that hash tables are not affected by this problem because the use of a hash function results in a random access pattern which leads to a low conflict probability. But of course, as a direct consequence of this randomization, hash tables do not support range scans.

\section{Evaluation}

For most experiments we used an Intel i5 4670T Haswell processor with 4 cores, $6 \mathrm{MB}$ shared L3 cache, and full HTM support through the Intel Transactional Synchronization Extensions. The maximum clock rate is $3.3 \mathrm{GHz}$, but can only be achieved when only one core is fully utilized. When utilizing all cores, we measured a sustained clock rate of $2.9 \mathrm{GHz}$.

By default, HyPer uses serial execution similar to VoltDB [1]; multiple threads are only used if the schema has been partitioned by human intervention. In the following we will call these execution modes serial and partitioned. Note that the partitioned mode used by HyPer (as in other systems) is somewhat cheating, since the partitioning scheme has to be explicitly provided by a human, and a good partitioning scheme is hard to find in general. In addition to these execution modes we included a $2 P L$ implementation, described in [21], as baseline for comparisons to standard database systems, and the hardware transactional memory approach (HTM) proposed here. We also include TSO with coarse-grained latches (optimistic) instead of HTM to show that TSO alone is not sufficient for good performance.

For most experiments we used the well-known TPC-C benchmark as basis (without "think times", the only deviation from the benchmark rules). We set the number of warehouses to 32 , and for the partitioning experiments the strategy was to partition both the data and the transactions by the main warehouse. We used the Adaptive Radix Tree [17] as index structure, although the scalability is similar with hash tables and red-black trees. In the following, we first look at scalability results for TPC-C and then study the interaction with HTM in microbenchmarks.

\subsection{TPC-C Results}

In a first experiment, we ran TPC-C and varied the number of threads up to the number of available cores. The results are shown in Figure 18 and reveal the following: First, classical $2 \mathrm{PL}$ is clearly inferior to all other approaches. Its overhead is too high, and it is even dominated by single-threaded serial execution. The latching-based optimistic approach has less overhead than 2PL, but does not scale because the coarsegrained (relation-level) latches severely limit concurrency. Both the partitioned scheme and HTM scale very well, with partitioning being slightly faster. But note that this is a comparison of a human-assisted approach with a fully automatic approach. Furthermore, the partitioning approach works so well only because TPC-C is "embarrassingly partitionable" in this low MPL setup, as we will see in the next experiment.

The reason that partitioning copes well with TPC-C is that most transactions stay within a single partition. By default, about $11 \%$ of all transactions cross partition boundaries (and therefore require serial execution to prevent collisions in a lock-free system). The performance depends crucially on the ability of transactions to stay within one partition. As shown in Figure 19, varying the percentage of partition-crossing transactions has a very deteriorating effect on the partitioning 


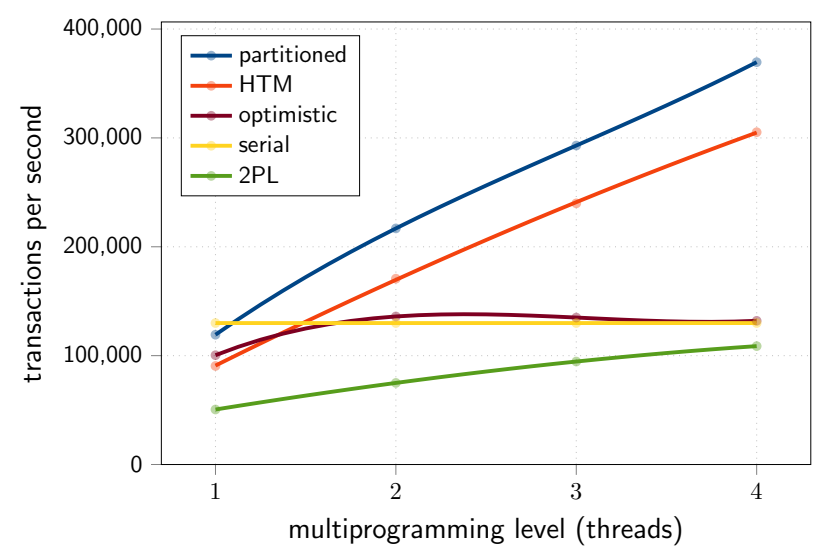

Figure 18. Scalability of TPC-C on desktop system

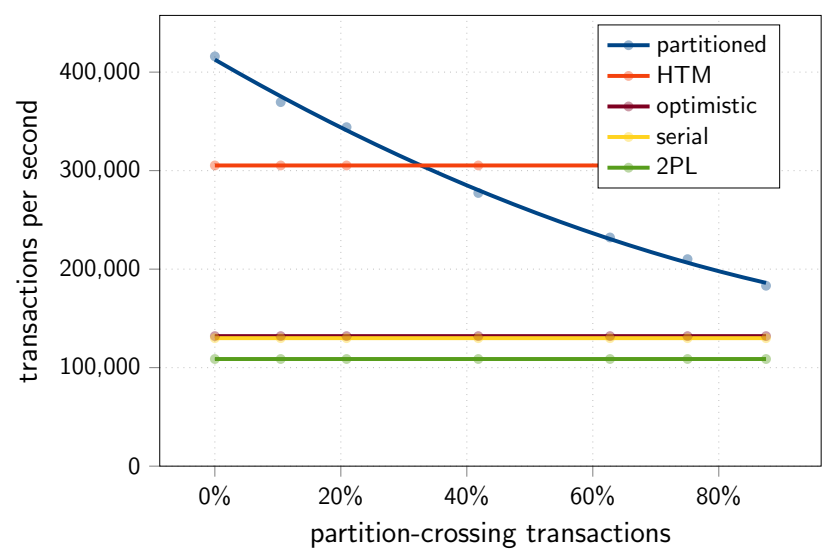

Figure 19. TPC-C with modified partition-crossing rates

approach, while the other transaction managers are largely unaffected because, in the case of TPC-C, partition-crossing does not mean conflicting. Therefore, picking the right partitioning scheme would be absolutely crucial; however, it is often hard to do-in particular if transactions are added to the workload dynamically.

Figure 20 shows results for TPC-C on the 28-core system described in Section 3. To reduce the frequent write conflicts, which occur in TPC-C at high thread counts, we set the number of warehouses to 200 , the number of insert zones to 64 , and the number of restarts to 30 . With these settings, our HTM-supported concurrency control scheme achieves a speedup of $15 \times$ and around 1 million TPC-C transactions per second with 28 threads. The other concurrency control schemes show similar performance and scalability characteristics as on the 4-core system. The figure also shows the performance of the Silo system [26] which we measured by using the publicly available source code that includes a handcoded TPC-C implementation in C++. Silo shows very good scalability, even at high thread counts, but is about $3 \mathrm{x}$ slower than HTM-supported HyPer with single-threaded execution.

\subsection{Microbenchmarks}

Our transaction manager was designed to be lightweight. Nevertheless, there is some overhead in comparison with an

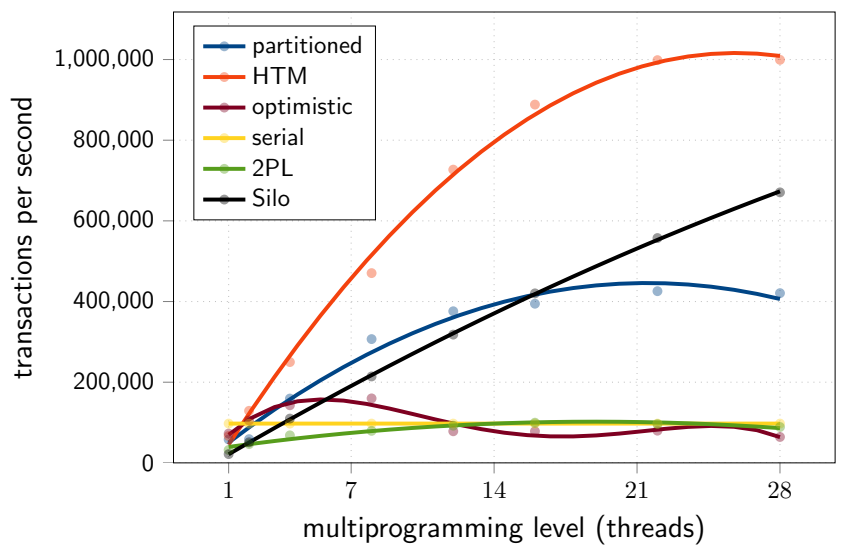

Figure 20. Scalability of TPC-C on server system

unsynchronized, purely single-threaded implementation. We determined the overhead by running the TPC-C benchmark using only one thread and enabling each feature separately: The HTM-friendly memory layout, including zone segmentation (with 8 zones), added 5\% overhead, mostly because of reduced cache locality. The HLE spinlocks, which are acquired for each tuple access, added 7\% overhead. Checking and updating the timestamps, slowed down execution by $10 \%$. Finally, transaction management, e.g., determining the safe timestamp, the transaction ID, etc. caused $7 \%$ overhead. In total, these changes amounted to a slowdown of $29 \%$. HyPer compiles transactions to very efficient machine code, so any additional work will have noticeable impact. However, this is much lower than the overhead of the 2PL implementation, which is $61 \%$ ! And of course the overhead is completely paid off by the much superior scalability of the HTM approach.

One interesting question is if it would be possible to simply execute a database transaction as one large HTM transaction. To analyze this, we used binary instrumentation of the generated transaction code to record the read and write sets of all TPC-C transactions. We found that only the delivery and order-status transactions have a cacheline footprint of less than $7 \mathrm{~KB}$ and could be executed as HTM transactions. The other transactions access between $18 \mathrm{~KB}$ and $61 \mathrm{~KB}$, and would usually exhaust the transactional buffer. Therefore, executing TPC-C transactions as monolithic HTM transactions is not possible. And other workloads will have transactions that are much larger than the relatively simple TPC-C transactions. Therefore, a mechanism like our timestamp scheme is required to cope with large transactions.

As we discussed in Section 4, there are two types of conflicts: timestamp conflicts and HTM conflicts. Timestamp conflicts must be handled by the transaction manager and usually result in a rollback of the transaction. We measured that $12 \%$ of all TPC-C transactions were aborted due to a timestamp conflict, but only $0.5 \%$ required more than 2 restarts. Most aborts occur at the warehouse relation, which has only 32 tuples but is updated frequently.

While HTM conflicts do not result in a rollback of the entire transaction, they result in the acquisition of relationlevel latches-greatly reducing concurrency. Using hardware 


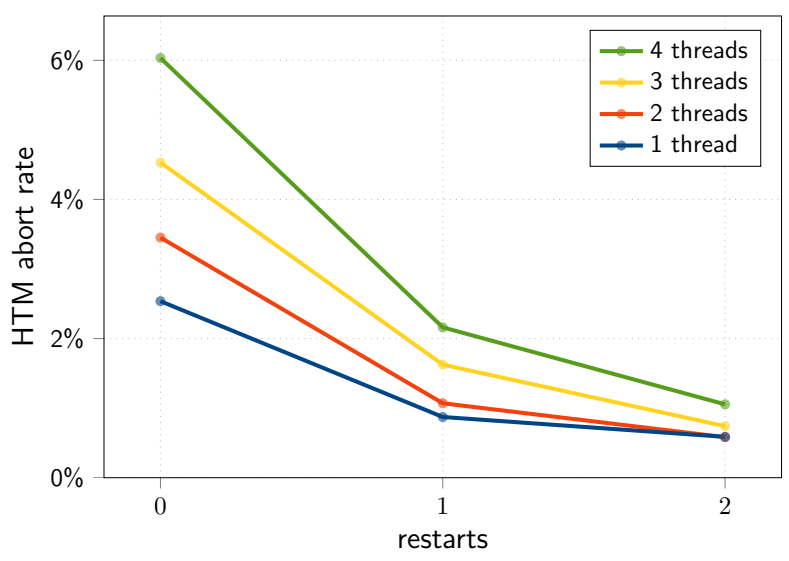

Figure 21. HTM abort rate with 8 declustered insert zones

counters, we measured the HLE abort rate of TPC-C, and found that $6 \%$ of all HLE transactions were aborted. This rate can be reduced by manually restarting transactions after abort by using Restricted Transaction Memory (RTM) instructions instead of HLE. As Figure 21 shows, the abort rate can be reduced greatly by restarting aborted transaction, i.e., most aborts are transient. With 4 threads, restarting has only a small positive effect on the overall transaction rate $(1.5 \%)$, because a $6 \%$ abort rate is still "small enough" for 4 threads.

These low abort rates are the outcome of our HTM-friendly storage layout from Section 5. Because TPC-C is very insertheave, with only one zone, the HLE abort rate rises from $6 \%$ to $14 \%$, and the clashes often do not vanish after restarts. Therefore, a careful data layout is absolutely mandatory to benefit from HTM. Note though that we did not decluster surrogate key generation, which makes conflicts even more unlikely, but would have required changes to the benchmark.

\section{Related Work}

Optimizing the transaction processing for modern multi-core and in-memory database systems is a vibrant topic within the database community. In the context of H-Store/VoltDB [7], [27] several approaches for automatically deriving a database partitioning scheme from the pre-defined workload were devised [28], [29] and methods of optimizing partition-crossing transactions were investigated [30]. The partitioning research focused on distributed databases, but is also applicable to shared memory systems. Partitioning the database allows for scalable serial transaction execution as long as the transactions do not cross partition boundaries, which in general is hard to achieve. In [31] a data-oriented transaction processing architecture is devised, where transactions move from one processing queue to another instead of being assigned to a single thread. The locking-based synchronization is optimized via speculative lock inheritance [32]. Ren et al. [33] found that the lock manager is a critical performance bottleneck for main memory database systems. They propose a more lightweight scheme, where, instead of locks in a global lock manager data structure, each tuple has two counters that indicate how many transactions requested read or write access. In an earlier eval- uation we showed that timestamp-based concurrency control has become a promising alternative to traditional locking [34].

Tu et al. [26] recently designed an in-memory OLTP system that uses optimistic concurrency control and a novel B-Tree variant [35] optimized for concurrent access. Lomet et al. [36] and Larson et al. [8] devised multi-version concurrency control schemes that, like our approach, use a timestamp-based version control to determine conflicting operations. Unlike our proposal, their concurrency control is fully software-implemented, therefore it bears some similarity to software transactional memory [37].

Herlihy and Moss [6], [38] proposed HTM for lock-free concurrent data structures. Shavit and Touitou [39] are credited for the first STM proposal. A comprehensive account on transactional memory is given in the book by Larus and Rajwar [40]. Due to the entirely software-controlled validation overhead, STM found little resonance in the database systems community - while, fueled by the emergence of the now common many-core processors, it was a vibrant research activity in the parallel computing community [41].

Wang et al. [42] combine Haswell's HTM with optimistic concurrency control to build a scalable in-memory database systems. Their approach similar to ours, but requires a final commit phase that is executed in a single hardware transaction and which encompasses the meta data of the transactions' read and write set. Karnagel et al. [43] performed a careful evaluation of HTM for synchronizing B-Tree access. Litz et al. [44] use multi-versioning, an old idea from the database community, to speed up TM in hardware.

\section{Summary and Future WORK}

There are two developments-one from the hardware vendors, and one from the database software developers- that appear like a perfect match: the emergence of hardware transactional memory (HTM) in modern processors, and main-memory database systems. The data access times of these systems are so short that the concurrency control overhead, in particular for locking/latching, is substantial and can be optimized by carefully designing HTM-supported transaction management. Even though transactions in main-memory databases are often of short duration, the limitations of HTM's read/write set management precludes a one-to-one mapping of DBMS transactions to HTM transactions.

We therefore devised and evaluated a transaction management scheme that transforms a (larger) database transaction into a sequence of more elementary, single tuple access/update HTM transactions. Our approach relies on the well-known timestamp ordering technique to "glue" the sequence of HTM transactions into an atomic and isolated database transaction. Our quantitative evaluation on a mainstream Haswell processor showed that our approach has low overhead and excellent scalability.

In future work we will investigate our approach on other hardware platforms with HTM like IBM's Power8.

\section{ACKNOWLEDGMENTS}

We thank Konrad Lai for insightful discussions, and Markus Widmer for arranging early access to the Haswell systems. 


\section{References}

[1] M. Stonebraker and A. Weisberg, "The VoltDB main memory DBMS," IEEE Data Eng. Bull., vol. 36, no. 2, 2013.

[2] A. Kemper and T. Neumann, "HyPer: A hybrid OLTP\&OLAP main memory database system based on virtual memory snapshots," in ICDE, 2011.

[3] F. Färber, S. K. Cha, J. Primsch, C. Bornhövd, S. Sigg, and W. Lehner, "SAP HANA database: data management for modern business applications," SIGMOD Record, vol. 40, no. 4, 2011.

[4] J. Lindström, V. Raatikka, J. Ruuth, P. Soini, and K. Vakkila, "IBM solidDB: In-memory database optimized for extreme speed and availability," IEEE Data Eng. Bull., vol. 36, no. 2, 2013.

[5] P.-Å. Larson, M. Zwilling, , and K. Farlee, "The Hekaton memoryoptimized OLTP engine," IEEE Data Eng. Bull., vol. 36, no. 2, 2013.

[6] M. Herlihy and J. E. B. Moss, "Transactional memory: Architectural support for lock-free data structures," in ISCA, 1993.

[7] S. Harizopoulos, D. J. Abadi, S. Madden, and M. Stonebraker, "OLTP through the looking glass, and what we found there," in SIGMOD, 2008.

[8] P.-Å. Larson, S. Blanas, C. Diaconu, C. Freedman, J. M. Patel, and M. Zwilling, "High-performance concurrency control mechanisms for main-memory databases," PVLDB, vol. 5, no. 4, 2011.

[9] V. Leis, A. Kemper, and T. Neumann, "Exploiting hardware transactional memory in main-memory databases," in ICDE, 2014.

[10] J. Gray and A. Reuter, Transaction Processing: Concepts and Techniques. Morgan Kaufmann, 1993.

[11] C. Cascaval, C. Blundell, M. M. Michael, H. W. Cain, P. Wu, S. Chiras, and S. Chatterjee, "Software transactional memory: why is it only a research toy?" Commun. ACM, vol. 51, no. 11, 2008.

[12] "Intel architecture instruction set extensions programming reference," http://software.intel.com/file/41417, 2013.

[13] A. Wang, M. Gaudet, P. Wu, J. N. Amaral, M. Ohmacht, C. Barton, R. Silvera, and M. M. Michael, "Evaluation of Blue Gene/Q hardware support for transactional memories," in PACT, 2012.

[14] C. Jacobi, T. J. Slegel, and D. F. Greiner, "Transactional memory architecture and implementation for IBM system z," in MICRO, 2012.

[15] R. Rajwar and M. Dixon, "Intel transactional synchronization extensions," http://intel.com/go/idfsessions, 2012.

[16] J. L. Hennessy and D. A. Patterson, Computer Architecture - A Quantitative Approach (5. ed.). Morgan Kaufmann, 2012.

[17] V. Leis, A. Kemper, and T. Neumann, "The adaptive radix tree: ARTful indexing for main-memory databases," in ICDE, 2013.

[18] J. J. Levandoski, D. B. Lomet, and S. Sengupta, "The Bw-tree: A B-tree for new hardware platforms," in ICDE, 2013.

[19] N. Diegues and P. Romano, "Self-tuning intel transactional synchronization extensions," in ICAC, 2014.

[20] N. Narula, C. Cutler, E. Kohler, and R. Morris, "Phase reconciliation for contended in-memory transactions," in OSDI, 2014.

[21] H. Mühe, A. Kemper, and T. Neumann, "Executing long-running transactions in synchronization-free main memory database systems," in $C I D R, 2013$.

[22] M. J. Carey, "Modeling and evaluation of database concurrency control algorithms," Ph.D. dissertation, 1983

[23] P. A. Bernstein, V. Hadzilacos, and N. Goodman, Concurrency Control and Recovery in Database Systems. Addison-Wesley, 1987.

[24] C. Mohan, D. Haderle, B. Lindsay, H. Pirahesh, and P. Schwarz, "ARIES: a transaction recovery method supporting fine-granularity locking and partial rollbacks using write-ahead logging," ACM Trans. Database Syst., vol. 17, no. 1, 1992.

[25] P. Felber, C. Fetzer, P. Marlier, and T. Riegel, "Time-based software transactional memory," IEEE Trans. Parallel Distrib. Syst., vol. 21, no. $12,2010$.

[26] S. Tu, W. Zheng, E. Kohler, B. Liskov, and S. Madden, "Speedy transactions in multicore in-memory databases," in SOSP, 2013

[27] E. Jones and A. Pavlo, "A specialized architecture for high-throughput OLTP applications," HPTS, 2009.

[28] C. Curino, Y. Zhang, E. P. C. Jones, and S. Madden, "Schism: a workload-driven approach to database replication and partitioning," PVLDB, vol. 3, no. 1, 2010.

[29] A. Pavlo, C. Curino, and S. B. Zdonik, "Skew-aware automatic database partitioning in shared-nothing, parallel OLTP systems," in SIGMOD, 2012.

[30] E. P. C. Jones, D. J. Abadi, and S. Madden, "Low overhead concurrency control for partitioned main memory databases," in SIGMOD, 2010.

[31] I. Pandis, R. Johnson, N. Hardavellas, and A. Ailamaki, "Data-oriented transaction execution," $P V L D B$, vol. 3, no. 1, 2010.
[32] R. Johnson, I. Pandis, and A. Ailamaki, "Improving OLTP scalability using speculative lock inheritance," $P V L D B$, vol. 2, no. 1, 2009.

[33] K. Ren, A. Thomson, and D. J. Abadi, "Lightweight locking for main memory database systems," PVLDB, vol. 6, no. 2, 2013.

[34] S. Wolf, H. Mühe, A. Kemper, and T. Neumann, "An evaluation of strict timestamp ordering concurrency control for main-memory database systems," in IMDM, 2013.

[35] Y. Mao, E. Kohler, and R. T. Morris, "Cache craftiness for fast multicore key-value storage." in EuroSys, 2012.

[36] D. B. Lomet, A. Fekete, R. Wang, and P. Ward, "Multi-version concurrency via timestamp range conflict management," in ICDE, 2012.

[37] D. Dice, O. Shalev, and N. Shavit, "Transactional locking II," in DISC, 2006.

[38] M. Herlihy, "Fun with hardware transactional memory," in SIGMOD, 2014.

[39] N. Shavit and D. Touitou, "Software transactional memory," in PODC, 1995.

[40] J. R. Larus and R. Rajwar, Transactional Memory. Morgan \& Claypool Publishers, 2006.

[41] M. Herlihy and N. Shavit, The art of multiprocessor programming. Morgan Kaufmann, 2008.

[42] Z. Wang, H. Qian, J. Li, and H. Chen, "Using restricted transactional memory to build a scalable in-memory database," in EuroSys 2014, 2014.

[43] T. Karnagel, R. Dementiev, R. Rajwar, K. Lai, T. Legler, B. Schlegel, and $\mathrm{W}$. Lehner, "Improving in-memory database index performance with intel ${ }^{\circledR}$ transactional synchronization extensions," in HPCA, 2014.

[44] H. Litz, D. R. Cheriton, A. Firoozshahian, O. Azizi, and J. P. Stevenson, "SI-TM: reducing transactional memory abort rates through snapshot isolation," in ASPLOS, 2014.

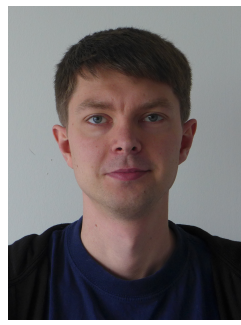

Viktor Leis is a PhD student in the database group at TUM. His research revolves around the HyPer main-memory database system. In particular, he focuses on optimizing HyPer for modern hardware. Viktor Leis holds a MSc in Computer Science from TUM and an undergraduate degree in business information systems from Regensburg University of Applied Sciences.

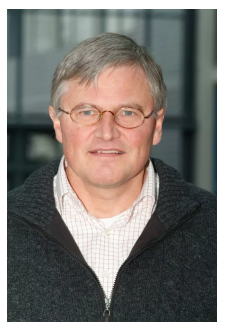

Alfons Kemper studied Computer Science at the University of Dortmund, Germany, and the University of Southern California, Los Angeles, where he obtained the Ph.D. in 1984. He had been on the faculty at the Technical University of Karlsruhe, the Technical University RWTH Aachen, the University of Passau before assuming the Chair professorship for Database Systems at the Technische Universität München (TUM) in 2004. His current research interests focus on scalable information management systems, in particular main-memory database system concepts. Together with his colleague Thomas Neumann he initiated and leads the HyPer main-memory project at TUM.

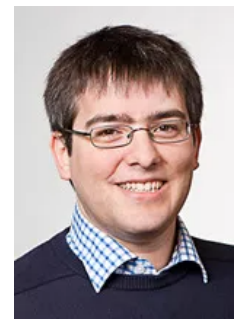

Thomas Neumann conducts research on database systems, focusing on query optimization and query processing. As part of that research he has build two very successful systems, RDF-3X for efficient processing of large RDF data, and the very fast main-memory database system HyPer. Their development induced many innovative techniques, including advanced join ordering techniques and efficient query compilation approaches. He studied business information systems at the University of Mannheim and received a doctorate in informatics from the same university. Before joining TUM as professor, Neumann was a senior researcher at the Max Planck Institute for Informatics. 